\title{
Pflegefachkraft, medizinische Fachangestellte, Rettungsassistent \& Co. - Was passiert, wenn etwas schief geht?
}

Tobias Weimer

(C) Springer-Verlag Wien 2012

Personalnot allerorts - ob nun in der Stationspflege im OP oder auch der ambulanten Pflege, überall wird händeringend nach qualifizierten Mitarbeitern gesucht. Dazu kommt der wirtschaftliche Zwang zur Erlösoptimierung im DRG-Zeitalter. Kein Wunder, dass viele Arbeitgeber längst ein Auge auf fachfremde Kräfte werfen. Vom Rettungsassistenten bzw. Notfallassistenten, über die Arzthelferin, dem Lagerungshelfer bis hin zur ungelernten Hilfskraft, viel scheint verbreitet zu sein; selbst der Hochrisikobereich „OP“ ist betroffen. Was ist sinnvoll, zulässig, was verantwortungslos?

Unter dem Gesichtspunkt der Qualität und einer möglichen Gefahrgeneigtheit einzelner oder regelhaft auf Hilfskräfte übertragener Tätigkeiten sind auch unter dem Blickwinkel eines möglichen Organisationsverschuldens folgende Fragen zu beantworten: Wen und wie viele Hilfskräfte ziehe ich für welche Tätigkeit (z. B. medizinische Assistenz, Behandlungspflege, Lagerung, Hol- und Bringedienst etc.) heran? Ist die übertragene Tätigkeit für den Patienten gefährlich, setzt die Durchführung der Maßnahme spezielles ärztliches bzw. fachpflegerisches Sonderwissen voraus? Werden die Fachkräfte (ärztliches und/oder pflegerisches Personal) mit Anleitungs-, Beratungs- und Supervisionsaufgaben möglicherweise überlastet?

Werden diese Kontrollfragen verantwortungsbewusst beantwortet, so wird im Regelfall das Haftungsrisiko begrenzt sein. Anderenfalls setzen sich alle Beteiligten einem nicht unbeträchtlichen straf- wie zivilrechtlichen Haftungsrisiko aus, sollte der Patient durch die Hilfskraft schuldhaft an seinem Körper oder in seiner Gesundheit geschädigt werden. So haftet die Hilfskraft ggf. persönlich nach den Grundsätzen des Übernahmeverschuldens. Aber auch die Einrichtung selbst sowie der jeweilige Delegationsverantwortliche haftet im Schadensfalle zivilrechtlich nach den Grundsätzen der Delegationsverantwortung und des Organisationsverschuldens. Die Einrichtung ist nämlich aufgrund des geschlossenen Behandlungs-Pflegevertrages dazu verpflichtet, den Patienten ordnungsgemäß zu behandeln bzw. zu pflegen. Insbesondere besteht die Verpflichtung, den Patienten vor Gefahrenquellen zu schützen, die regelmäßig aus der Risikosphäre der Einrichtung und ihres Personals resultieren. Verstößt die Einrichtung bzw. ihr Personal schuldhaft gegen diese Pflicht, ist sie zivilrechtlich zum Schadensersatz und Schmerzensgeld verpflichtet. Das Verschulden des jeweils behandelnden Pflegepersonals oder des behandelnden Arztes wird dem Krankenhaus bzw. Pflegeeinrichtungsträger zugerechnet, da diese als seine Erfüllungsgehilfen handeln. Strafrechtlich steht eine fahrlässige Körperverletzung (vgl. § 229 StGB) oder gar Tötung (vgl. $\S 222 \mathrm{StGB}$ ) im Raum.

T. Weimer $(\bowtie)$

WEIMER BORK Kanzlei für Medizin- und Strafrecht,

Bochum, Deutschland 